\title{
Perspectives of Semantic Web in E- Commerce
}

\author{
B. VijayaLakshmi \\ M.Tech (CSE), \\ KIET,
}

\author{
A.GauthamiLatha \\ Dept. of CSE, \\ VIIT,
}

\author{
Dr. Y. Srinivas \\ Dept. of IT, \\ GITAM University,
}

\author{
Mr. K.Rajesh \\ Dept. of MCA, \\ KIET,
}

\begin{abstract}
The Semantic Web Technologies growing interest in human computer interactions where it enables the machines to interpret the data published in a machine interpretable form under web. Currently, E-Commerce is seriously vulnerable due to the lack of proper standards where HTML does not provide the syntax and semantics of information. Semantic Web has the potential to extremely influence the further development of the internet market, where e-commerce plays an important role. This paper discusses the semantic architecture for e-commerce using ontology language like RDF. This gives the overview about how the producer describes their resource in ontology content and how the consumer will retrieve the efficient data.
\end{abstract}

\section{Keywords}

E-Commerce, RDF, Semantic Web, Ontology.

\section{INTRODUCTION}

With the development of World Wide Web more companies are updating their data in the web. In traditional web the search method for data is not only tedious and time consuming, but also results in many unrelated information. The reason is, due to various data accessing methods and data description used in the traditional web. The semantic web overcomes all these drawbacks. Semantic web is not the replacement of current web but it is an extension of current web, in which information is given in a well defined manner, so that it enable the computer and people to work in cooperation. This paper mainly focuses on the data description (Producer) and data accessing (Consumer) in e-commerce circumstances. In section 2, related terminologies and limitations in the current e-commerce are discussed. Section 3 introduces the architecture of semantic e-commerce application and the case study is explained in section 4. Finally the conclusion is given.

\section{RELATED TERMINOLOGY}

\subsection{E-Commerce}

Electronic Commerce, commonly Known as e-Commerce or ecommerce, consists of buying and selling the products or services over the internet. With the rapid development of internet and the number of goods online, the e-Commerce plays the important role in the present trends [1]. There are two major types emerged like Business-to-Consumer (B2C), and Business-toBusiness (B2B) e-commerce models.

The Business-to-Consumer (B2C) e-commerce model is a publicly accessible Web site offering products for sale to the customers. The Business-to-Business (B2B) e-commerce model specifically designed for organizations to conduct business over the Internet. In this paper we are concentrating only on B2C ecommerce.

Current E-Commerce: A search for any product or product offers is the starting point for most e-Commerce transactions. ECommerce web applications are designed to return the most appropriate data to the user but the current applications are failing in returning the relevant data to the consumers.

\section{Limitations in the Current $E$-Commerce are}

Information Asymmetry \& Price Dispersion: This situation occurs where the same product with same features is available with different price values in different websites to the consumers [1].

Semantic Description \& Extension is Deficient: This situation occurs where the product's generic attributes are not considered, such as price, color, function, origin and material etc...

Business Attributes: This situation occurs where the customers choose the tax percentage, type of pay and discount offered if any etc...[3]

Interoperability in an inconsistent environment: This situation occurs where the consumer is in the conflicting state to choose the best option from the available websites.

For example: Let's take a case of specific search for "'mobile phone' camera email download ring tones",

Case1: In current web search, it will not certainly return a list containing all mobile phones with a camera, email function and the ability to download ring tones. It may either display the list of mobiles with camera and also without camera or else it simply displays about either only different camera models or different ring tones.

Case2: we have several websites that sell the same product at different prices. A consumer, who is new to online purchasing will default opts the Amazon site and buy the product for $\$ 301.99$. A consumer who has much experience in online purchasing will search various web sites and finally gets the product for \$250.11.So the total savings for the experienced consumer is $\$ 51.88$. Hence there is a significant gain due to the information asymmetry, this is price dispersion. if we spend much time for the search we can buy the better product. Figl shows the curve by comparing the time and benefit of search. 


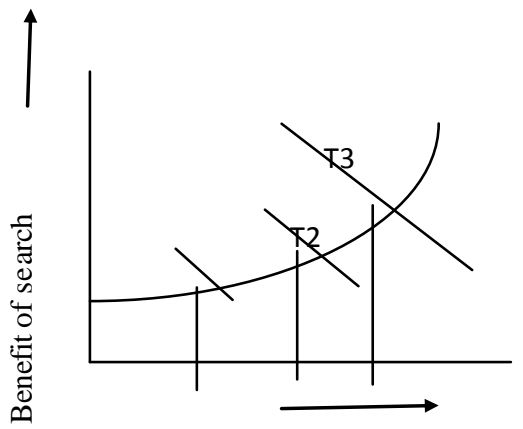

Time spent

\section{Fig 1: Search \& time curve}

\subsection{Semantic Web}

The initiation for the Semantic Web was by Sir Tim Berners Lee, who is the famous inventor of WWW and one of the W3C directors. Semantic Web is an extension of the current web, in which the semantics of information and the services of the web is defined, making it possible for the web to understand and satisfy the requirements of the people to use the web content $[9,15]$

With a Semantic Web, we can easily handle many issues, like knowledge-repository, search agent, information parser, etc., which cannot be done well with the current web $[9,15]$. To make the web semantic, we need new standard web ontology languages. Ontology is a key to the Semantic Web. Ontology's are used to express information in a machine interpretable form.

\subsubsection{Ontology}

Ontology is a set of specifications, relationships and constraints that describe a certain domain [4]. It defines the common terms needed to share information relevant to that domain. Ontology plays the key role in agent communication as it improves the accuracy of searching and enables the development of powerful applications that tackle complicated questions, whose answers do not reside on a single web page.

Some basic ontology languages are RDF (Resource Description Framework) and OWL (Web Ontology Language) [10]. In this paper we concentrate only on RDF. Some development editors are altova [17], protégé [16], ontolingna, UNSPSC, Rosetta Net.

RDF (Resource Description Framework): $\quad \mathrm{RDF}$ is a language for expressing data models in XML syntax. XML provides an elemental syntax to structure the data [7]. It provides the meaning to that structured data $[14,15]$. RDF is used to describe web resources. RDF assertion consists of a triplet (subject, predicate, object) in which a subject has a property that property value can be either a string literal or a reference to another resource.

For example: Let us consider the Car and its Properties.

The mileage of the car is $15 \mathrm{kmph}$ (or)

The color of the car is gray.

Here, the subject is car

The predicates can be either mileage or color
And the objects can be are $15 \mathrm{kmph}$ or gray

The syntactic representation is as follows:

$<$ Subject, predicate, object>

$<$ Car, color, gray > or

$<$ Car, mileage, $15 \mathrm{kmpk}>$

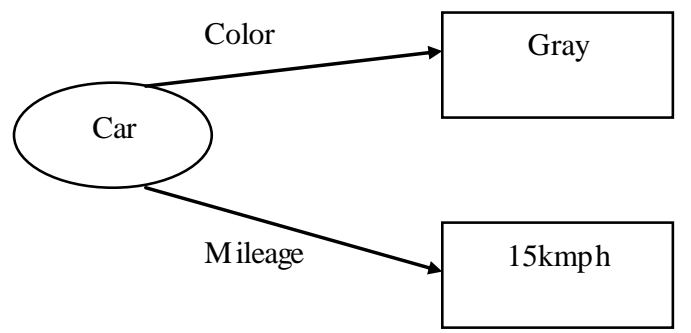

Fig 2: Example RDF graph

The Comparisons between Traditional web and semantic web with some parameters [6] is given in table 1 .

Table 1: Traditional web Vs Semantic web

\begin{tabular}{|c|c|c|}
\hline Comparison & $\begin{array}{c}\text { Traditional Web } \\
\text { Service }\end{array}$ & Semantic Web Service \\
\hline Service & Simple & Compounded \\
\hline Service Requester & People & Computer \\
\hline Service Description & Words based & Ontology based \\
\hline Data Access & Syntax based & Semantic based \\
\hline
\end{tabular}

\section{SEMANTIC WEB E-COMMERCE} ARCHITECTURE

In the previous section we have explained the current ecommerce and its drawbacks and also stated that the Semantic web application can overcome these drawbacks. Fig. 3 shows the architecture of the semantic web based e-commerce application. 


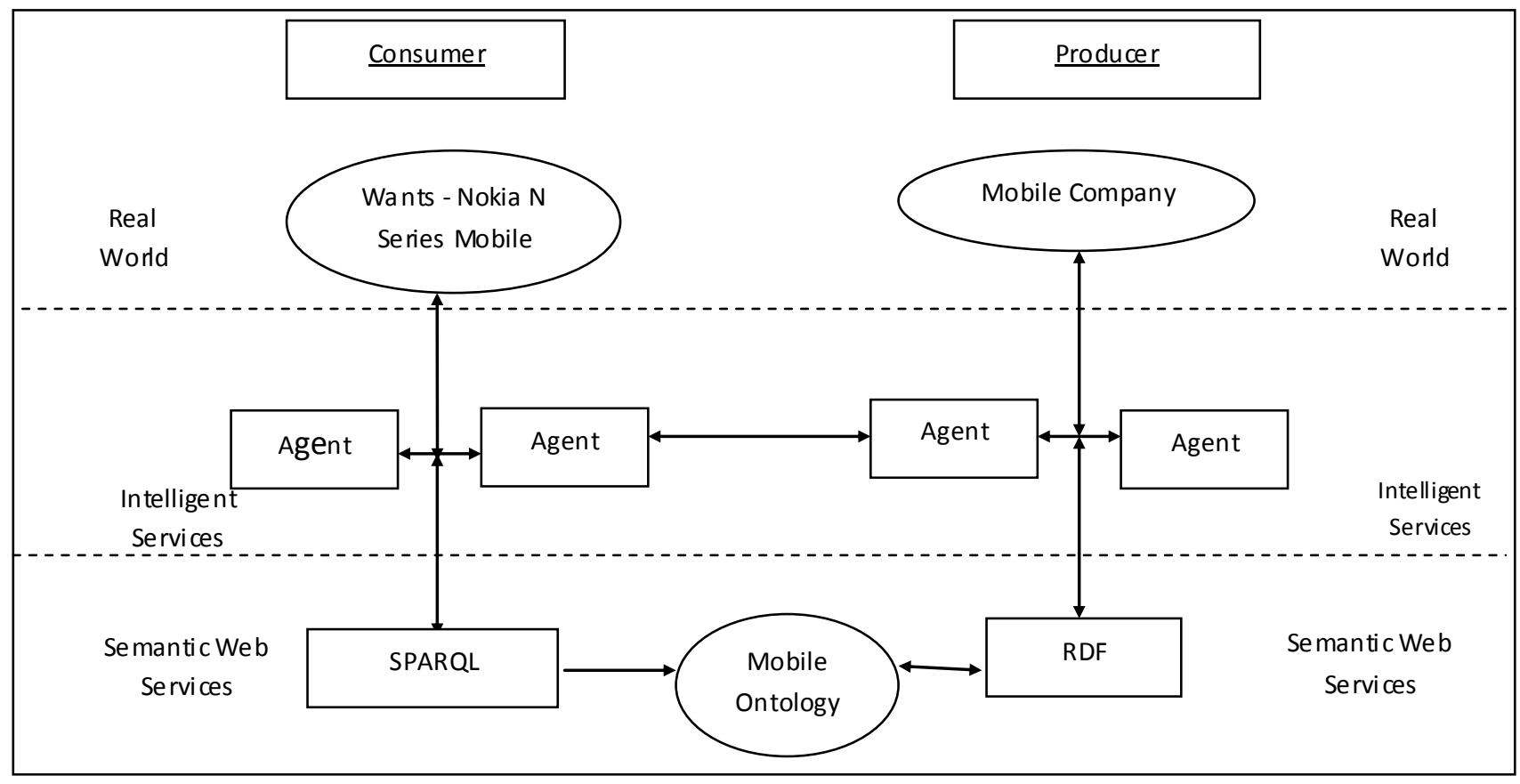

Fig 3: Architecture of Semantic Web E-Commerce

Here, Producer is the one, who manufactures the products and concentrates on the quality and efficiency of the particular product and advertise the details in the web market. Consumer is an individual who buys products or services for personal use over the internet.

Agents are meant to reduce the consumer's work and information overload [2]. In the increasing growth of e-commerce technology, services and information available on the internet, agents plays a very important role. Agents are active, autonomous and personalized software's to which tasks can be delegated.

In architecture, we have two types of agents.

\section{Search agent}

\section{Ontology agent}

The consumer directly communicates with search agent, who is responsible for retrieving the metadata of documents. Here we used the SPARQL query language to retrieve the related information to the consumers. The Producer communicates with ontology agent, who provides the knowledge of ontology to answer queries about the domain and its structure. Here we use the Jena framework [11] to build the ontology like RDF.

From the architecture it has shown that, any product or service should be described ontologically to retrieve the result in semantic manner. For example, the mobile company provides the related terms and structures related to that domain and the mobile database to the agent. The agent generates the RDF. Whenever the consumer or user want to search the information that information is passed to the search agent; the search agent searches the related information using SPARQL query lan guage [5]

\section{CASE STUDY}

Let us take the mobiles; in this case the ontology agent will create the RDF for mobiles. Using Jena the RDF generated is represented in the table 2 .

Table 2: Mobiles RDF

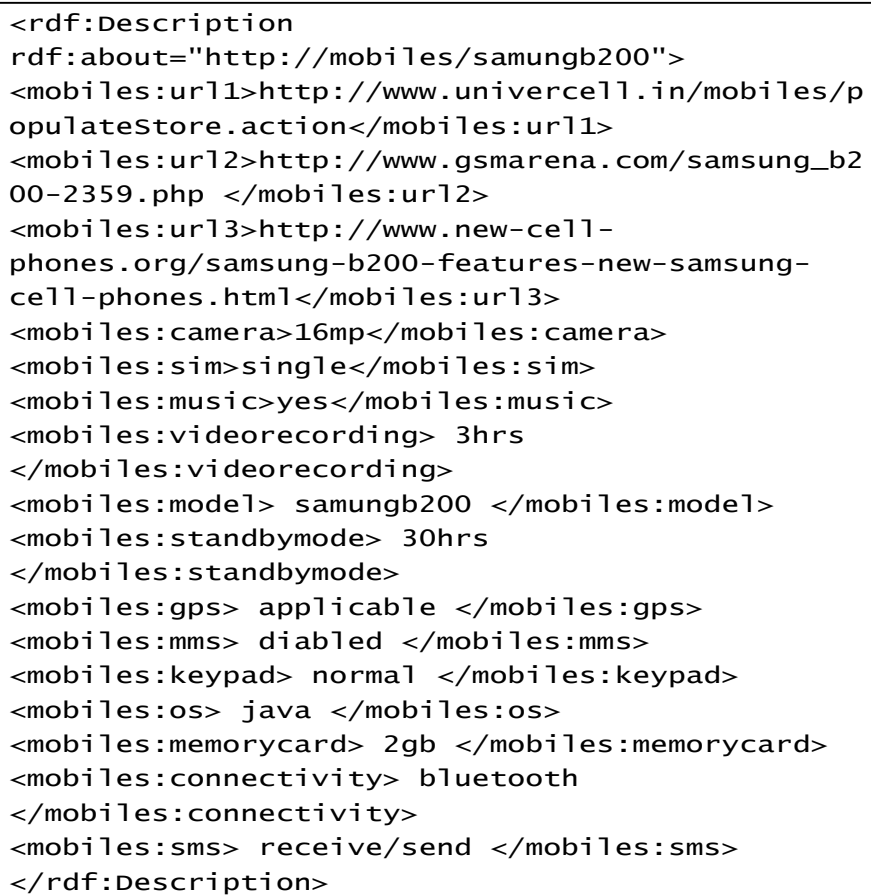


The RDF in table 2 describes the features of mobile. As section 2.2.1 shows the syntax of RDF, the mobile RDF also represents their features in the format -
$<$ Subject, predicate, object>. Like

<mobiles: standby mode>30hrs</mobiles: standby mode> <mobiles: gps>applicable </mobiles: gps>

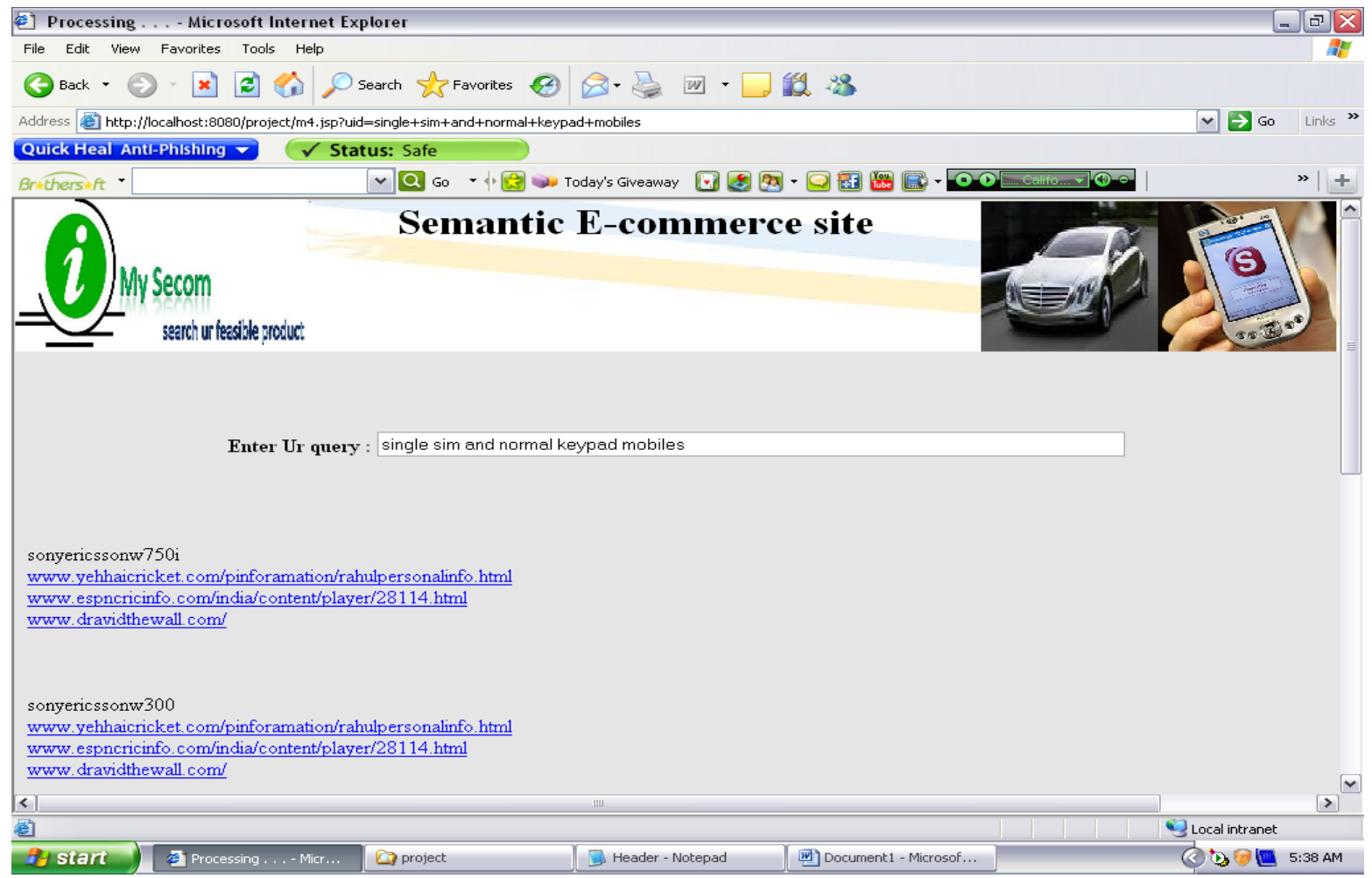

Fig. 4: Screen shot of semantice-commerce user interface

In the above screen shot the consumer passes the query "single sim and normal keypad mobiles". To generate the result the search agent searches this information using sparql query [5]. The query is shown in table 3 .

Table 3: SPARQL Query

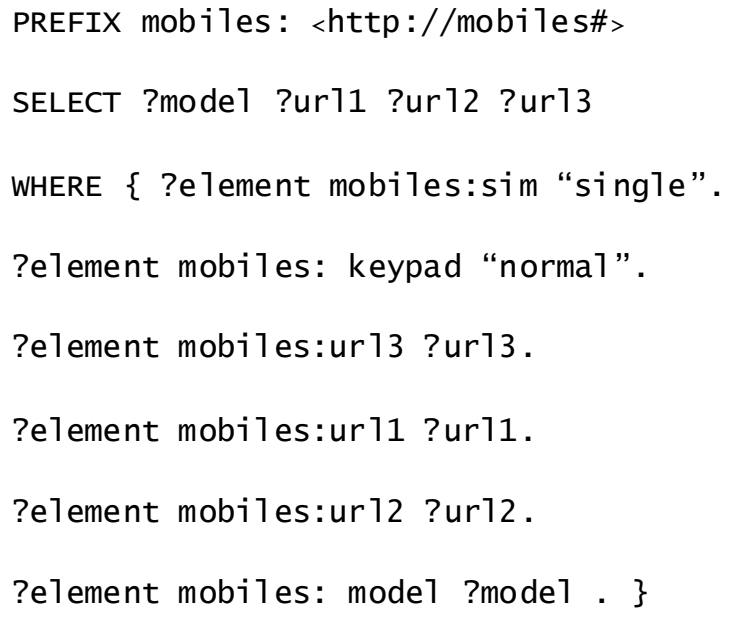

\section{CONCLUSION}

There is a great demand for retrieving and sharing the information across the internet in an efficient way. Semantic web and software agents are providing direction in this regard. This paper introduces a semantic web based e-commerce application which is suitable for retrieving the data without inconsistency of data. Semantic web technology also offers various services like automatic producer recognition, automatic product and service recognition and in price and quality comparisons.

\section{REFERENCES}

[1] Karim Heidari, "The Impact of Semantic Web on ECommerce", World Academy of Science, Engineering and Technology 512009

[2] Oscar Martinez, Federico Botella, "Building E-Commerce Web Applications: Agent- and Ontology-based Interface Adaptivity”, Operations Research Center, University Miguel Hernández of Elche, Avda.Universidad, s/n, 03202 Elche, Spain

[3] Donglin Chen Xiaofei Li Jun Zhang, "User-oriented intelligent service of e-catalog based on semantic web ", Information Management and Engineering (ICIME), 2010 The 2nd IEEE International Conference. 
[4] Rasheed M. Al-Zahrani, "The Ontology problem in ecommerce applications”, Information SystemsDept., KSU.

[5] SPARQL Query language for RDF http://www.w3.org/TR/rdfsparql-query/

[6] Tanja Sollazzo, Siegfried Handschuh, Steffen Staab, Martin Frank, Nenad Stojanovic, "Semantic Web Service Architecture -Evolving Web Service Standards toward the Semantic Web" FLAIRS-02 Proceedings.

[7] Resource Description Framework (RDF Semantic web standards www.w3.org/RDF/

[8] Peter mika "Social networks and the semantic web".

[9] Tim Berners-Lee "Semantic Web on XML" slides:http://www.w3.org/2000/Talks/1206-xml2k-tbl

[10] Grigoris Antoniou and Frank van Harmelen "Semantic web Primer".
[11] Jena RDF API http://jena.sourceforge.net/tutorial/RDF_API/index.html

[12] Tanya Gupta, Abir Qasem,"Reduction of price dispersion through Semantic E-commerce: A Position Paper". Semantic Web Workshop 2002 Hawaii, USA

[13] John Davies, Rudi Studer, Paul Warren "Semantic web Technologies- trends and research in ontology based systems".

[14] Sergey Melnik: Bridging the Gap between RDF and XML. December1999.http://www.db.stanford.edu/ melnik/rdf/fusi on.html

[15] Tim Berners-Lee: Why RDF model is different from the XML model. Design Issues draft, October 1998

[16] The Protégé Ontology Editor and Knowledge Acquisition System protege.stanford.edu/

[17] Altova editor www.altova.com 